\title{
Oscillatory decay at the Rosensweig instability: Experiment and theory
}

\author{
Bert Reimann, ${ }^{1, *}$ Reinhard Richter, ${ }^{1}$ Ingo Rehberg, ${ }^{1}$ and Adrian Lange ${ }^{2}$ \\ ${ }^{1}$ Experimentalphysik V, Universität Bayreuth, D-95440 Bayreuth, Germany \\ ${ }^{2}$ Institut für Theoretische Physik, Universität Magdeburg, D-39106 Magdeburg, Germany
}

(Received 22 May 2003; published 30 September 2003)

\begin{abstract}
Transient patterns of the Rosensweig instability are accessed with a pulse sequence. The critical scaling behavior of the oscillation frequency and of the propagation velocity of these patterns is experimentally investigated by switching the magnetic induction to subcritical values. The experimental findings are in good agreement with the linear theory, if the low viscosity and the finite thickness of the magnetic liquid layer are taken into account. In this way we elucidate the subcritical branch of the underlying steady state bifurcation, which is situated in the immediate vicinity of a splitting type bifurcation.
\end{abstract}

DOI: 10.1103/PhysRevE.68.036220

PACS number(s): 82.40.Bj, 47.20.Ma, 47.54.+r, 47.20.Ky

\section{INTRODUCTION}

Pattern formation has mostly been investigated in systems driven out of thermodynamic equilibrium, e.g., in RayleighBénard convection or Taylor-Couette systems [1]. On the contrary, examples for pattern formation in systems at equilibrium are buckled shell structures $[2,3]$ and the instability of a horizontal fluid interface in a vertical electric [4] or magnetic field [5]. Here dissipation does only have an influence in the transient process of structure formation, not at its final state. For sufficiently low dissipation these systems are close to the ideal, Hamiltonian case, and thus exhibit interesting bifurcation scenarios.

From an experimental point of view, the normal field instability in a magnetic field is conveniently accessible. In 1967 Cowley and Rosensweig first investigated the influence of a homogeneous magnetic field on a horizontally extended layer of magnetic fluid (MF). When surpassing a critical value $B_{c}$ of the magnetic induction, they observed a hysteretic transition between the flat surface and a hexagonal pattern of liquid crests $[5,6]$.

In the present paper we are investigating the fate of the pattern when switching to a subcritical induction. In particular, the oscillatory decay of magnetic liquid ridges $[7,8]$ towards a flat surface is observed. It turns out that the oscillation frequency and the propagation velocity of the decaying pattern depend sensitively on the subcritical magnetic induction. When approaching the bifurcation point $B_{\mathrm{c}}$ from below, a dramatic decrease of both observables can be measured.

We examine this bifurcation point theoretically within the framework of a linear theory. The critical scaling behavior is discussed particularly in the context of a perturbation of the so called $[9,10]$ splitting type bifurcation. Here a pair of conjugated complex eigenvalues with negative real parts, equivalent of an oscillatory decay, merges at the negative real axis just before the origin of the complex plane. At least one of the eigenvalues propagates on the positive real axis, equivalent of a developing pattern. In the limit of vanishing viscosity the theory predicts a splitting type bifurcation.

\footnotetext{
*Present address: Fraunhofer Institute for Factory Operation and Automation, D-39106 Magdeburg, Germany.
}

The paper is organized as follows. After a sketch of the experimental setup in Sec. II, we present in Sec. III the measurements of the oscillation frequency and the propagation velocity of the ridges for different magnetic induction. The theoretical context is displayed in Sec. IV with an emphasis on the set of solutions of the dispersion relation for supercritical and subcritical induction. Section V is devoted to the discussion and the conclusion of our results.

\section{EXPERIMENTAL SETUP}

Our experimental setup is shown in Fig. 1. A cylindrical vessel with an edge machined from Teflon ${ }^{\circledR}$ with a diameter of $12 \mathrm{~cm}$ and a depth of $3 \mathrm{~mm}(2 \mathrm{~mm})$ is brimful filled with fluid and is situated in the center of a pair of Helmholtz coils. The experiments are performed with the magnetic fluid EMG 909 (Ferrotec Corp.). The fluid is illuminated from above by 90 red light emitting diodes mounted on a ring of $30 \mathrm{~cm}$ diameter. This ring is placed $105 \mathrm{~cm}$ above the surface. A charge-coupled-device camera is positioned in the center of the ring. By this construction, a flat surface reflects no light into the camera lens. Only an inclined surface of proper angle will reflect light into the camera. The camera is connected via a frame grabber to a 90-MHz Pentium PC and serves additionally as the fundamental clock for timing the experiment.

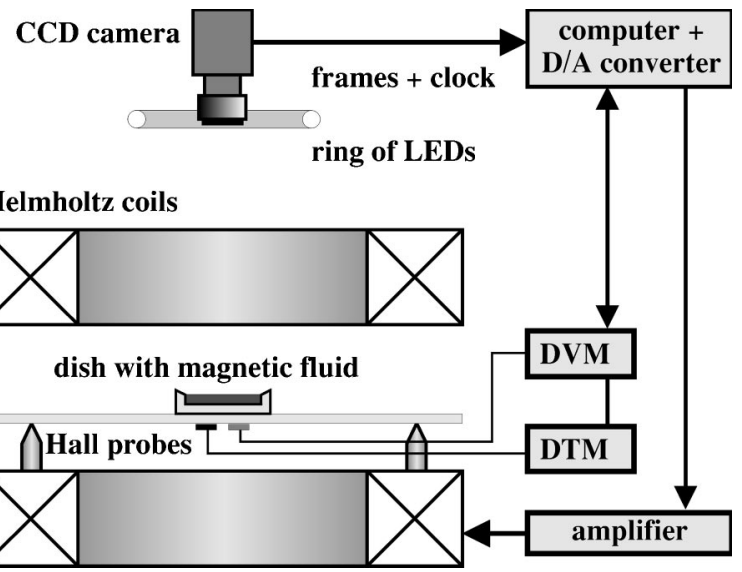

FIG. 1. Scheme of the experimental setup. 


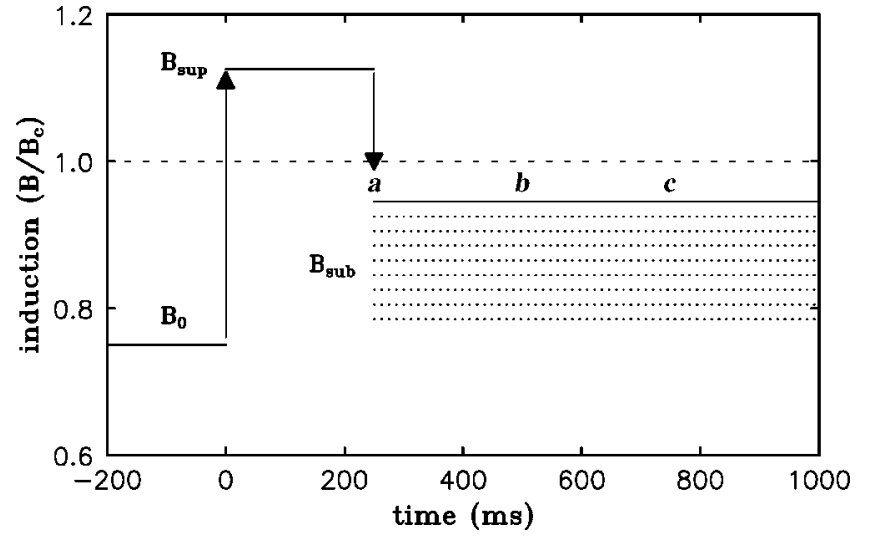

FIG. 2. Pulse sequence for the preparation of the liquid ridges. The small letters $a, b, c$ mark the times when the pictures of Fig. 3 have been captured.

In order to prepare a state of unstable liquid ridges, the magnetic induction has to be switched in a jumplike manner [7]. We start all measurements from a common subcritical induction $B_{0}=12.75 \mathrm{mT}$. The jumplike increase of the induction is initiated by the computer. Its digital-to-analog converter is connected via an amplifier (fug Elektronik $\mathrm{GmbH}$ ) to the Helmholtz coils (Oswald Magnetfeldtechnik). The magnet system cannot follow the control signal instantly; its relaxation time $\tau_{B}$ to a jumplike increase of the control signal depends on the jump $\Delta B$. For a maximal jump of $\Delta B$ $=7 \mathrm{mT}$, the relaxation time mounts up to $\tau_{B}=8.0 \mathrm{~ms}$. The other characteristic time scales of the system are the capillary time scale, $t_{c}=\sigma^{1 / 4} /\left(g^{3 / 4} \rho^{1 / 4}\right) \simeq 12.5 \mathrm{~ms}$, and the viscous time scale, $t_{\nu}=\sigma /(g \mu) \simeq 450.3 \mathrm{~ms}$. The dynamic viscosity $\nu$ and surface tension with air $(\sigma)$ of the MF are taken from the literature [11]. The density $\rho$ of the MF is measured to be $1.16 \mathrm{~g} / \mathrm{cm}^{3}$, which is somewhat higher than the value of $1.02 \mathrm{~g} / \mathrm{cm}^{3}$ given by the producer. The gravitational acceleration is denoted by $g$. For the empty Helmholtz coils, the spatial homogeneity of the magnetic field is better than $\pm 1 \%$. This grade is valid within a cylinder of $10 \mathrm{~cm}$ diameter and $14 \mathrm{~cm}$ in height oriented symmetrically around the center of the coils. Two Hall probes are positioned immediately under the vessel. A Siemens Hall probe (KSY 44) serves to measure the magnetic field during the jumplike increase, and is connected via a digital voltmeter (Prema 6001). For measuring a constant magnetic field and for calibration purposes, we use a commercial Hall probe (Group3LPT-231) connected to the digital teslameter (DTM 141). Both devices are controlled via an IEEE bus by the computer.

\section{EXPERIMENTAL RESULTS}

In the following we demonstrate the preparation of the state of liquid ridges. First the induction is increased jumplike from $B_{0}=12.75 \mathrm{mT}$ up to a supercritical value $B_{\text {sup }}$, as sketched in Fig. 2. Due to the supercritical induction, liquid ridges emerge. Before they start to develop into the hexagonal state, the induction is switched back to a subcritical value $B_{\text {sub }}>B_{0}$. Now the liquid ridges decay in an oscillatory man-
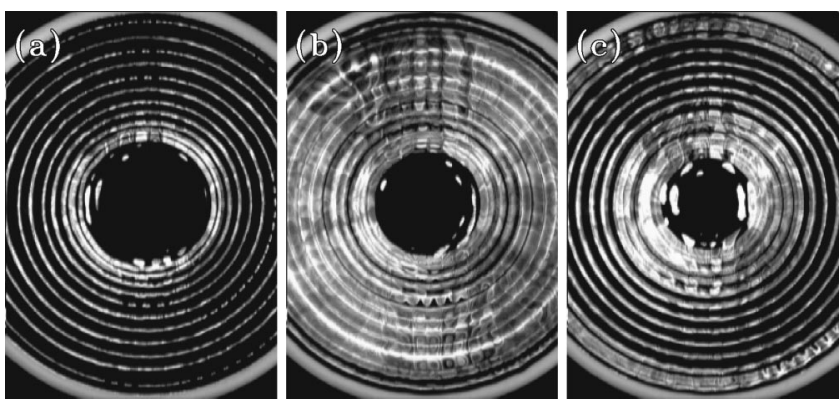

FIG. 3. Initial pattern of liquid ridges (a), approximately flat surface after a quarter of the oscillation period (b), and liquid ridges after half of the period (c).

ner. Figure 3 shows half of such an oscillation period. The oscillation starts with a pattern of concentric liquid ridges, as presented in Fig. 3(a). Figure 3(b) shows the approximately flat surface after a quarter of the period. The liquid ridges after half of the oscillation period are displayed in Fig. 3(c).

\section{A. Oscillation frequency}

Next we describe the extraction of the oscillation frequency. Therefore we calculate the azimuthal average of the gray level values in the vessel, as described in detail in Ref. [7], which defines a single horizontal line in the space-time plot of Fig. 4(a). These lines are displayed as a function of the delay time-indicated by the vertical axis-after switching to the subcritical value. In order to surpass the temporal resolution of the camera, the experiment has to be done repetitively with different delay times. Thus the space-time plot displays the evolution of the inclined (dark) and approximately horizontal (bright) parts of the surface of the magnetic liquid. The dashed line (i) denotes the start of the oscillation, when the magnetic induction was switched to the subcritical value. The gray value distribution is approximately repeated at the site of line (ii) in Fig. 4(a).
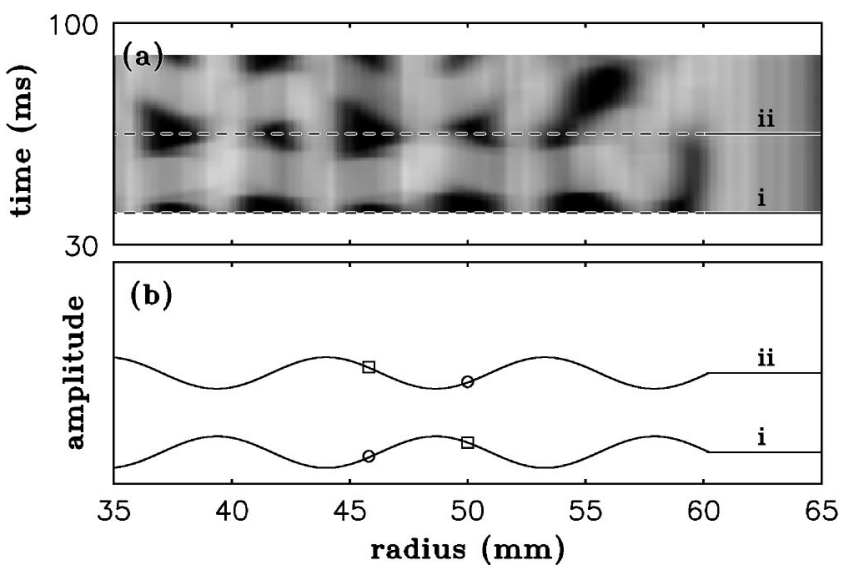

FIG. 4. (a) Space-time plot of the oscillation of the liquid ridges in the outer region of the vessel. The dashed horizontal line at i (ii) denotes the start (half of the period) of the oscillation, respectively. In (b) a sketch of the height profiles at the corresponding times is given. The circles and squares mark points of equal inclination. For clarity the height profiles have been vertically shifted. 

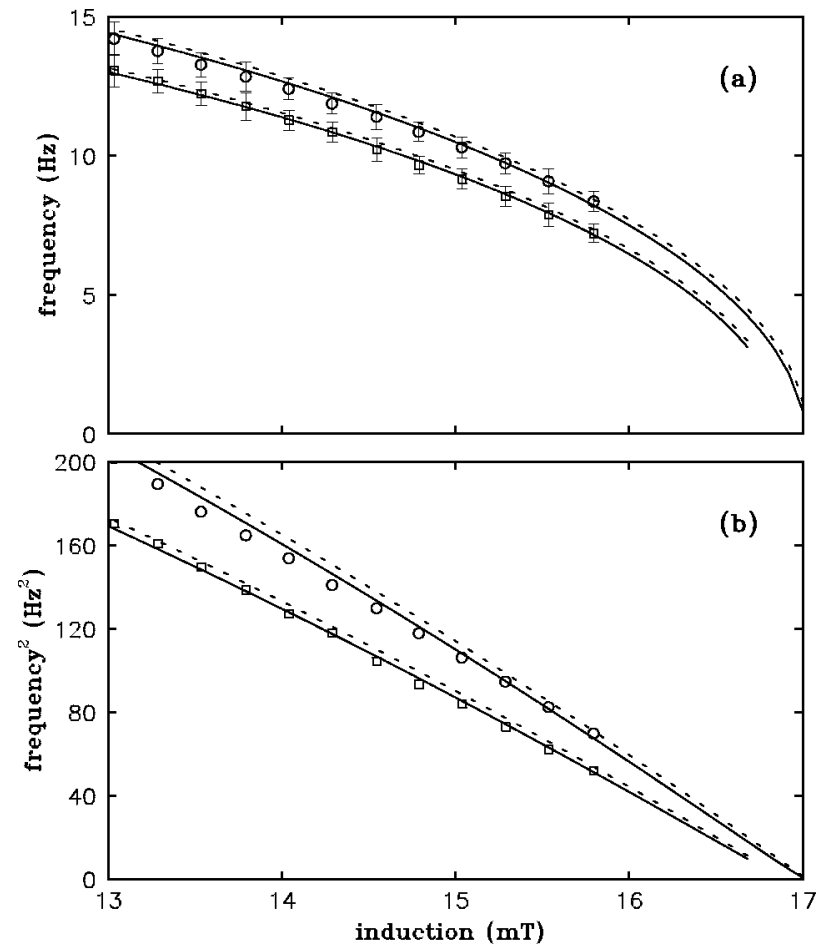

FIG. 5. (a) Oscillation frequency of the liquid ridges versus the subcritical magnetic induction. The open circles (squares) denote the measured data obtained after a pulse of $B_{\text {sup }}=18 \mathrm{mT}(17.5 \mathrm{mT})$, respectively. A layer thickness of $3 \mathrm{~mm}$ was used. The solid (dashed) lines give the theory for a viscous (inviscid) MF, respectively, as presented in Sec. IV. (b) Square of the oscillation frequency of the liquid ridges as a function of the subcritical magnetic induction.

The gray value is a measure of the deviation from the horizontal surface. The flat surface both at the valley and the crest corresponds to white areas. The interpretation of the measurement in Fig. 4(a) is sketched in Fig. 4(b) in terms of height profiles. It illustrates the fact that only half an oscillation period elapses between line (i) and (ii) in Fig. 4(a).

In order to determine the oscillation period, we select two radial positions with a lateral distance of a half wave length. The corresponding equal intensities do appear after half of the oscillation period at these positions, as illustrated in Fig. 4(b) by the open circles and squares. This fairly complicated procedure turned out to be more practical than the simpler method of measuring the full period because of the fast decay of the patterns.

The oscillation period as a function of the magnetic induction is shown in Fig. 5. Starting with a jump to the supercritical induction $B_{\text {sup }}=18 \mathrm{mT}$, the oscillation period was measured for 12 different values of the subcritical induction $B_{\text {sub }}$. The measured data are denoted in Fig. 5(a) by open circles. The error bars are estimated from seven independent measurements. The data show a square-root-like dependence from the induction, and are well described by the theory (solid line), developed in Sec. IV. A second measurement series has been performed for $B_{\text {sup }}=17.5 \mathrm{mT}$. In this way, a pattern of liquid ridges with a smaller wave number has been prepared. The data for these oscillation frequencies are

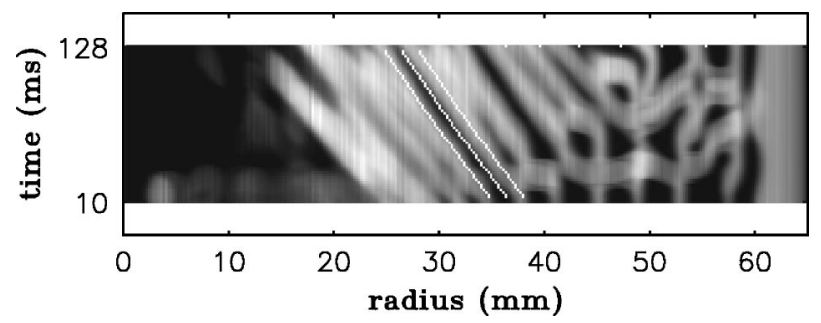

FIG. 6. Space-time plot of the propagation of the magnetic liquid ridges in a vessel of $2 \mathrm{~mm}$ depth. The three solid lines denote the ridge from which the propagation velocity has been extracted. The whole vessel is shown with its edge at the right hand side.

marked in Fig. 5 by open squares. Again a good agreement between experimental data and theory is found. In Fig. 5(b), the square of the oscillation frequency has been plotted versus the induction in order to show the range of validity of the square-root-like behavior.

\section{B. Propagation velocity}

After a jump to a subcritical magnetic induction the ridges do not only start to oscillate, but also have a finite propagation velocity. We have measured this behavior in a vessel of $2 \mathrm{~mm}$. Figure 6 shows the space-time plot of the gray value distribution of the whole vessel. In the right part, we observe the oscillatory behavior of the ridges already shown above and in the central part one can detect stripes with an inclination to the left, i.e., to the center of the vessel. These stripes stem from the ridges which are propagating towards the center of the vessel. The three white lines denote specific maxima and minima of the gray value distribution. Each line consists of 64 independent extrema. From a linear fit of the central white line, we estimate the propagation velocity of the ridge.

In Fig. 7(a), the propagation velocity obtained in this way is denoted by open squares and has been plotted versus the subcritical induction. Each data point has been estimated from six independent measurements. The data show a square-root-like behavior for a full decade of the velocity. In comparison with Fig. 5, this dependency could be corroborated even in the immediate vicinity of the bifurcation point. For comparison the open circles give the values for the propagation velocity as extracted from the data of the oscillation frequency in Fig. 5 by division with the wave number. The solid line gives the corresponding theoretical results for viscous fluids, a fluid layer of $3 \mathrm{~mm}$, and a magnetic permeability of $\mu_{\mathrm{r}}=1.94$ (see Sec. IV). The dotted line gives the theoretical results for a fluid layer of $2 \mathrm{~mm}$ and the same permeability, which does not seem to describe the measurements adequately. When considering the fact that the height is not necessarily constant when changing the magnetic field (as discussed in detail below), the height of the layer can be introduced as a fit parameter. The dash-dotted line represents the best fit yielding $h=1.04 \mathrm{~mm}$. It shows systematic deviations from the data. Thus in addition we use the magnetic permeability as a fit parameter, thus taking into account an aging of the magnetic fluid. The dashed line gives the best two-parameter fit, yielding $\mu_{\mathrm{r}}=1.98$ and $h=1.15 \mathrm{~mm}$. Fig- 


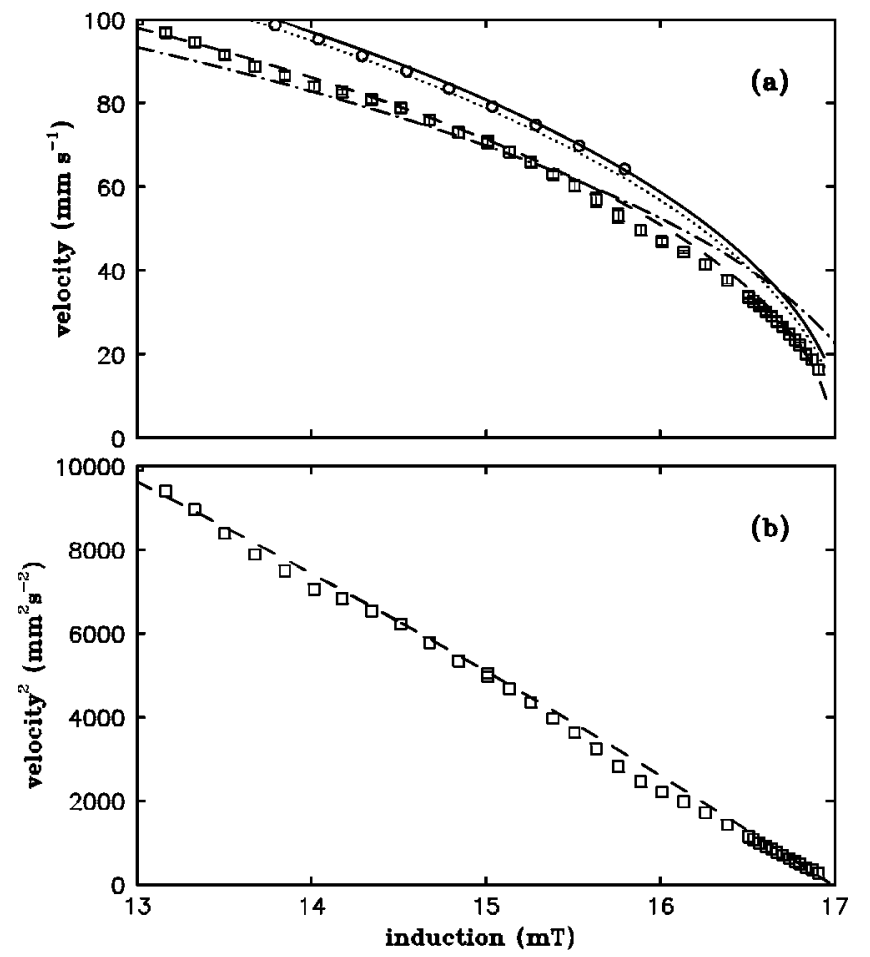

FIG. 7. (a) The open squares mark the propagation velocity of the liquid ridges measured as a function of the subcritical magnetic induction. For the measurements a preparing pulse of $B_{\text {sup }}$ $=18 \mathrm{mT}$ and a layer thickness of $2 \mathrm{~mm}$ were used. The open circles give the values for the propagation velocity as calculated from the oscillation frequencies of Fig. 5. The solid (dotted) line indicates the theoretical results for viscous fluids and a layer thickness of 3 $\mathrm{mm}(2 \mathrm{~mm})$, respectively (see Sec. IV). The dash-dotted line gives the best least squares fit by theory for keeping the permeability fixed at $\mu_{\mathrm{r}}=1.94$, where the height of the fluid layer, now used as a fit parameter, yields $h=1.04 \mathrm{~mm}$. The solid line gives the best twoparameter fit, where both, the magnetic permeability and thickness of the liquid layer have been used as fit parameters. The best fit has been obtained for $\mu_{\mathrm{r}}=1.98$ and $h=1.15 \mathrm{~mm}$. (b) Square of the propagation velocity versus the induction.

ure 7(b) shows the square of the velocity versus the subcritical induction.

\section{THEORY}

In the first part, we present those aspects of the linear theory which are necessary to calculate the measured scaling behavior, whereas the second part focuses on the peculiarities of the bifurcation scenario.

\section{A. Scaling behavior of the measured quantities}

For the sake of clarity in the presentation, the details of the method to calculate the above measured quantities are explained for an infinitely thick layer of magnetic fluid. A viscous MF of horizontally infinite extent subjected to a vertical magnetic induction is considered. The MF has a free surface with air above, where the basic state is that of a nondeformed surface. The dispersion relation for small disturbances from the basic state is given by [12]

$$
\begin{aligned}
& \left(1-\frac{i \omega}{2 \nu q^{2}}\right)^{2}+\frac{1}{4 \rho \nu^{2} q^{4}}\left[\rho g q+\sigma q^{3}-\frac{\left(\mu_{r}-1\right)^{2} B^{2} q^{2}}{\left(\mu_{r}+1\right) \mu_{0} \mu_{r}}\right] \\
& =\sqrt{1-\frac{i \omega}{\nu q^{2}}}
\end{aligned}
$$

where $\nu$ is the cinematic viscosity of the MF, $\mu_{\mathrm{r}}$ its relative permeability, $B$ the absolute value of the external magnetic induction, and $\mu_{0}$ the permeability of the vacuum. For dispersion relation (4.1), the small disturbances from the basic state were decomposed into normal modes, $\exp [-i(\omega t-\mathbf{q} \cdot \mathbf{r})]$. Here $\mathbf{r}=(x, y)$ is the planar space vector, $\mathbf{q}=\left(q_{x}, q_{y}\right)$ the wave vector, and $q=|\mathbf{q}|$ denotes the wave number. With $\omega=\omega_{1}+i \omega_{2}$, the real part of $-i \omega, \omega_{2}$, is called the growth rate and defines whether the disturbances will grow $\left(\omega_{2}>0\right)$ or decay $\left(\omega_{2}<0\right)$. The absolute value of the imaginary part of $-i \omega,\left|\omega_{1}\right|$, gives the angular frequency of the oscillation if it is different from zero. The critical induction for the onset of the instability reads as $B_{c}^{2}$ $=2 \mu_{0} \mu_{\mathrm{r}}\left(\mu_{\mathrm{r}}+1\right) \sqrt{\rho \sigma g} /\left(\mu_{\mathrm{r}}-1\right)^{2}[5]$.

For a supercritical induction $B_{\text {sup }}=1.05 B_{c}$, the dispersion relation (thick solid lines in Fig. 8) predicts that the linearly most unstable pattern evolves most quickly. It is characterized by the maximal growth rate $\omega_{\mathrm{m}}=i \omega_{2, \mathrm{~m}} \quad\left(\omega_{1, \mathrm{~m}} \equiv 0\right.$, $\left.\omega_{2, \mathrm{~m}}>0\right)$ and its corresponding wave number $q_{\mathrm{m}}$, marked by thin solid lines in Fig. 8(a). Such features can be concluded from the facts that at $q_{\mathrm{m}}$ the real part of $\omega^{2}$ has its smallest negative value and the imaginary part is zero.

According to the experimental procedure, the solution of the dispersion relation (4.1) for a subcritical induction at $q_{\mathrm{m}}$ is the relevant solution for the comparison with the experimental data. The thick dashed lines in Fig. 8 display the dispersion relation for $B_{\text {sub }}=0.96 B_{c}$. At the maximal wave number $q_{\mathrm{m}}$, one can read the relations $\operatorname{Re}\left(\omega^{2}\right)=\omega_{1}^{2}-\omega_{2}^{2}$ $>0$ and $\operatorname{Im}\left(\omega^{2}\right)=2 \omega_{1} \omega_{2}<0$ (thin dashed lines in Fig. 8). It is now difficult to conclude from these two relations whether the pattern decays, or grows and oscillates, respectively. Therefore it is advantageous for a viscous MF to plot instead the actual growth rate, $\operatorname{Re}(-i \omega)=\omega_{2}$, and the oscillation frequency, $\operatorname{Im}(-i \omega)=-\omega_{1}$, as shown in Fig. 9.

It has to be noted that in Fig. 8 for reasons of clarity only one solution of the dispersion relation for $B_{\text {sup }}$ and $B_{\text {sub }}$ is plotted. In the reduced interval of wave numbers in Fig. 9, all solutions are displayed. A detailed analysis about the number of solutions for dispersion relation (4.1) is presented in Ref. [13].

From Fig. 9, it becomes clear that the solution at $q_{\mathrm{m}}$ in the subcritical case corresponds to a decaying and oscillating pattern. The frequency of the oscillation is given by

$$
f=\frac{\left|\omega_{1}\right|}{2 \pi}
$$

and the experimentally measured propagation velocity by

$$
v=\frac{\left|\omega_{1}\right|}{q_{\mathrm{m}}} .
$$



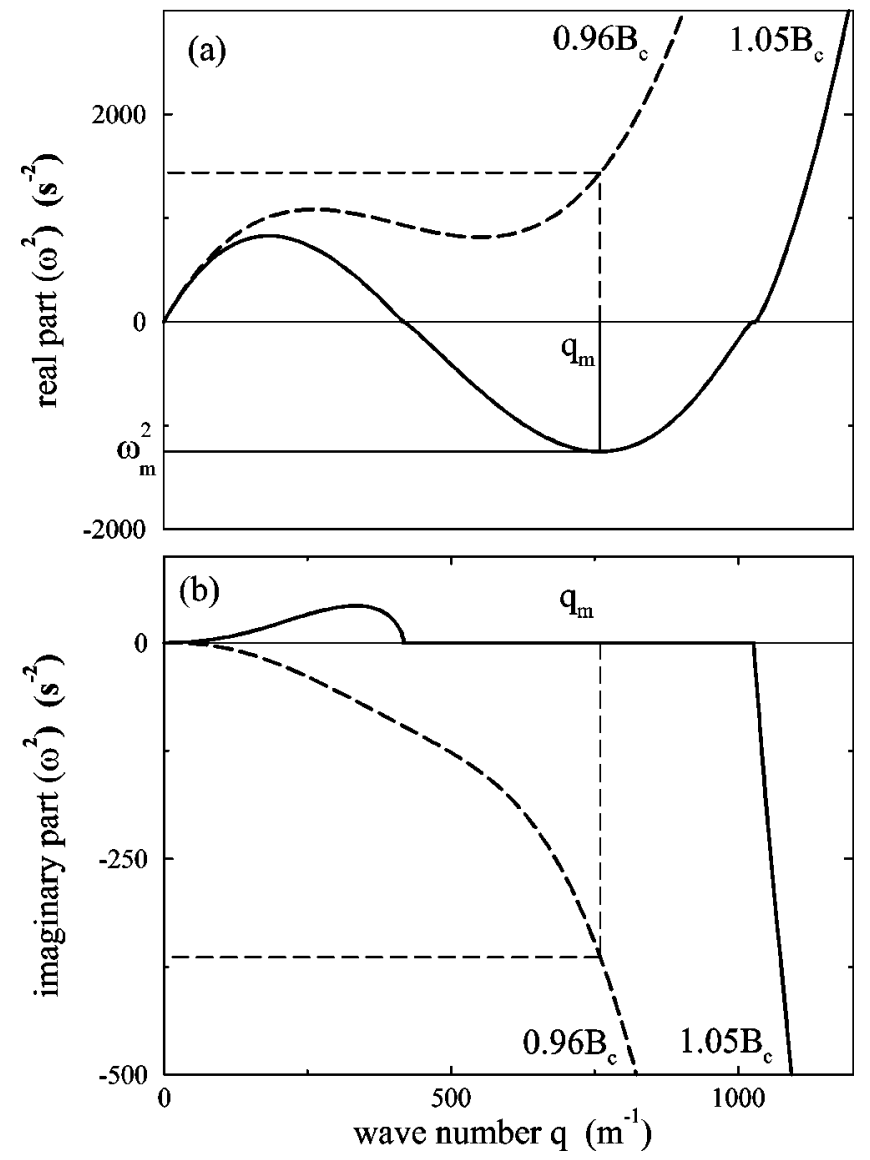

FIG. 8. Dependence of $\operatorname{Re}\left(\omega^{2}\right)$ (a) and $\operatorname{Im}\left(\omega^{2}\right)$ (b) on the wave number $q$ for a supercritical $\left(1.05 B_{c}\right.$, thick solid line) and a subcritical $\left(0.96 B_{c}\right.$, thick dashed line) induction. The wave number $q_{\mathrm{m}}$ of the linearly most unstable pattern and the corresponding value $\omega_{\mathrm{m}}^{2}$ are indicated by thin solid lines. The solution in the subcritical case for $q_{\mathrm{m}}$ is indicated by the thin dashed lines. The material parameters for the calculations are $\nu=5.17 \times 10^{-6} \mathrm{~m}^{2} / \mathrm{s}, \rho=1.16$ $\times 10^{3} \mathrm{~kg} / \mathrm{m}^{3}, \quad \sigma=2.65 \times 10^{-2} \mathrm{~kg} / \mathrm{s}^{2}, \quad \mu_{\mathrm{r}}=1.94, \quad$ and $B_{c}$ $=16.84 \mathrm{mT}$.

With the two formulas (4.2), (4.3), the frequency and the propagation velocity of the decaying pattern can be calculated and then compared with the experimental results. For this purpose, the dispersion relation in the case of a finite layer has to be applied [see Eq. (4.3) in Ref. [7]].

\section{B. Bifurcation}

Whereas in Fig. 9 the behavior of the growth rate and the oscillation frequency around $q_{\mathrm{m}}$ at a fixed subcritical induction is shown, the plot of $\omega_{2}$ and $-\omega_{1}$ versus the control parameter $B$, as shown in Fig. 10, reveals the influence of the finite viscosity on the bifurcation scenario.

For the limiting case of an inviscid fluid, the bifurcation diagram is given in Fig. 10 by dashed lines. The pattern oscillates below a certain threshold for the subcritical induction, $B_{\mathrm{c}, 0}\left[q_{\mathrm{m}}(\nu=0), \omega_{2}=0\right]$ [see Eq. (4.7)]. Above that threshold the pattern can either decay according to the solution $\omega_{2}<0$ or can develop towards the most unstable linear pattern belonging to $B_{\text {sub }}>B_{c}$ since $\omega_{2}>0$ is also a solution [14].
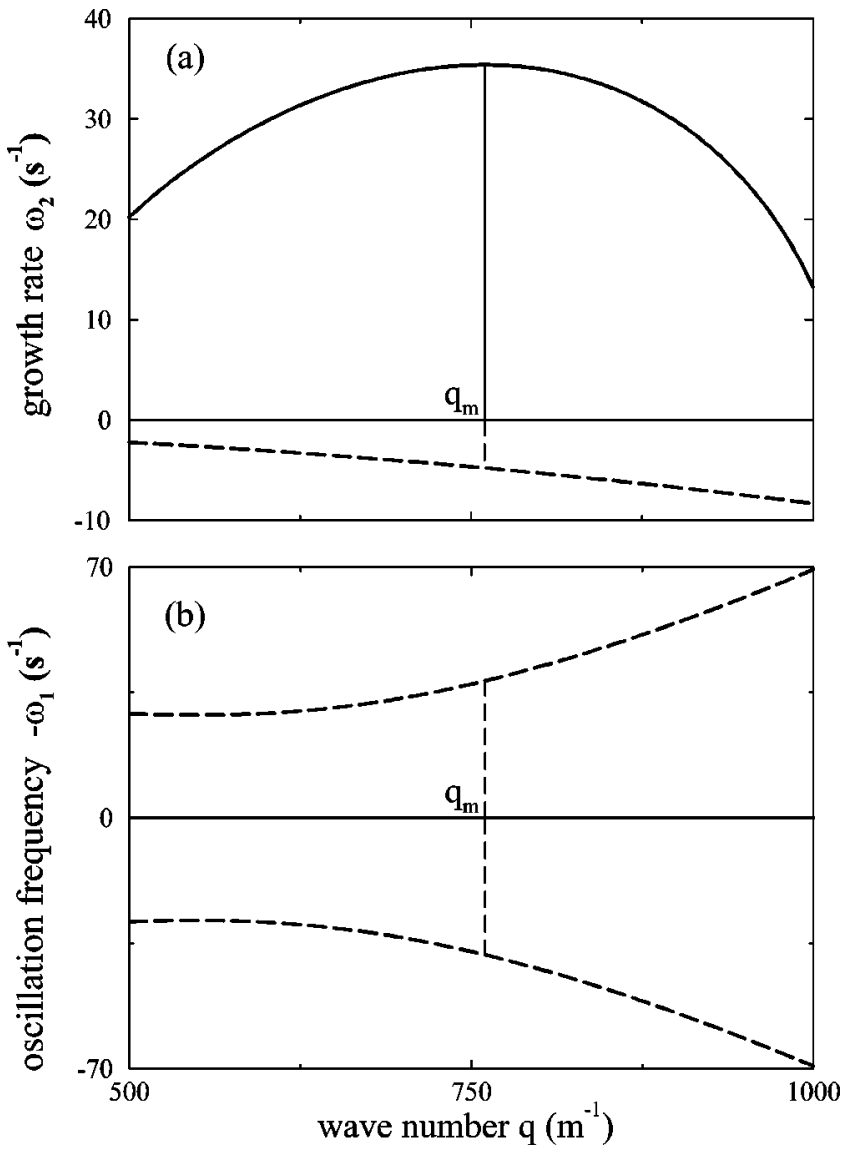

FIG. 9. Dependence of the growth rate $\omega_{2}$ (a) and the oscillation frequency $-\omega_{1}$ (b) on the wave number $q$ for the case of a supercritical (thick solid line) and a subcritical (thick dashed line) induction. The solutions at $q_{\mathrm{m}}$ for both cases are indicated by the corresponding thin lines. Parameters are the same as in Fig. 8.

For a viscous fluid (solid lines in Fig. 10) the behavior is more complex. A first critical induction $B_{\mathrm{c}, 1}$, occurs, where the set of solutions for dispersion relation (4.1) changes from two complex solutions to two negative real solutions. Both real solutions exist until at a second critical induction $B_{\mathrm{c}, 2}$, one of them abruptly ends, as explained below. At a third critical induction $B_{\mathrm{c}, 0}$, one of the two negative real solutions changes its sign and becomes positive. Note that $B_{\mathrm{c}, 0}$ does not give the onset of the Rosensweig instability from the flat surface, which takes place at $B_{c}<B_{c, 0}$. Rather $B_{\mathrm{c}, 0}$ characterizes the onset of growth of the preset pattern with wave number $q_{\mathrm{m}}$.

To understand this complex behavior, dispersion relation (4.1) is analyzed in dimensionless units (indicated by a bar) in the rearranged form

$$
\left(1+\frac{-i \bar{\omega}}{2 \bar{\nu} \bar{q}^{2}}\right)^{2}-\sqrt{1+\frac{-i \bar{\omega}}{\bar{\nu} \bar{q}^{2}}}=\frac{-\bar{q}-\bar{q}^{3}+2 \bar{B}^{2} \bar{q}^{2}}{4 \bar{\nu}^{2} \bar{q}^{4}} .
$$

All lengths were scaled with $[\sigma /(\rho g)]^{1 / 2}$, the time with $\sigma^{1 / 4} /\left(g^{3 / 4} \rho^{1 / 4}\right)$, the viscosity with $\sigma^{3 / 4} /\left(g^{1 / 4} \rho^{3 / 4}\right)$, and the induction with $B_{c}$. Equation (4.4) reveals that whatever value 

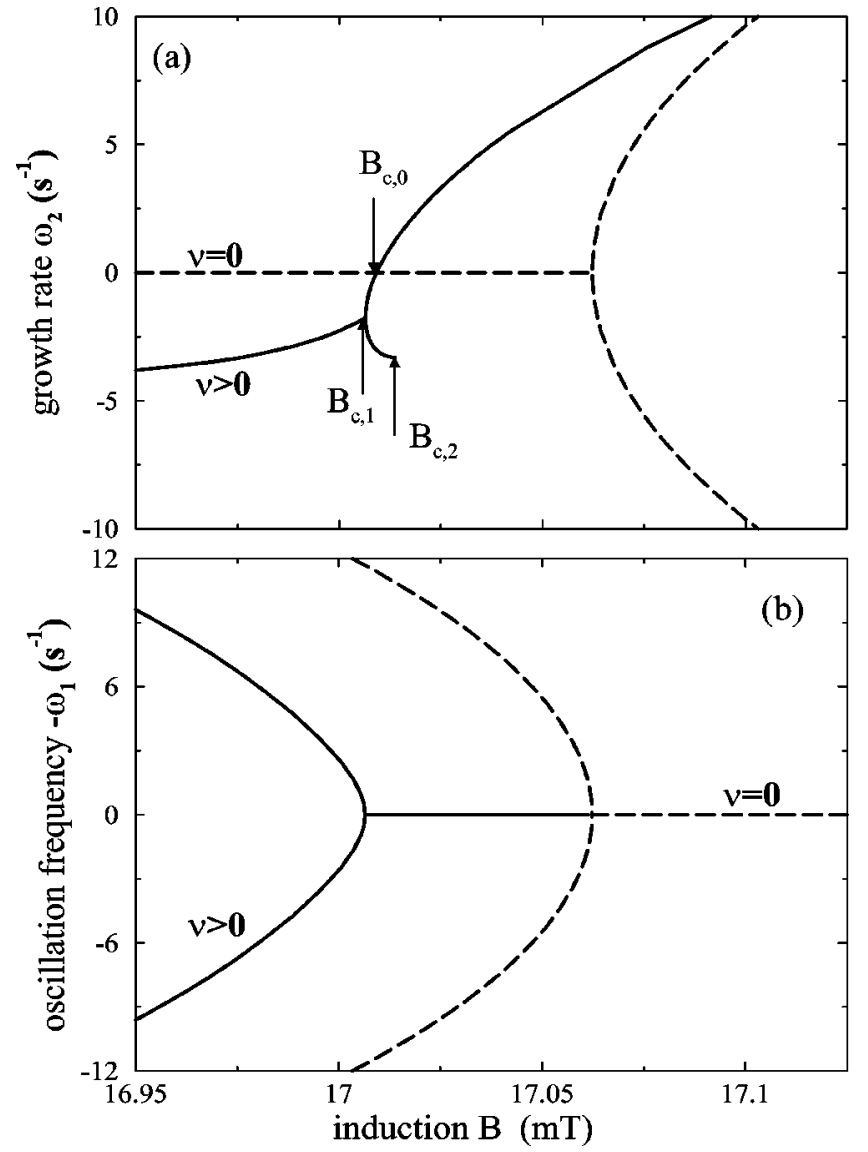

FIG. 10. Dependence of $\omega_{2}$ (a) and $-\omega_{1}$ (b) on the subcritical induction $B_{\text {sub }}$ for an inviscid MF ( $\nu=0$, dashed line) and a viscous MF ( $\nu=5.17 \times 10^{-6} \mathrm{~m}^{2} / \mathrm{s}$, solid line). The three critical inductions $B_{\mathrm{c}, 0}, B_{\mathrm{c}, 1}$, and $B_{\mathrm{c}, 2}$ are explained in the text. The remaining material parameters for the calculations are those from Fig. 8 with $q_{\mathrm{m}}(\nu)$ determined for $B_{\text {sup }}=18 \mathrm{mT}$.

$\bar{\omega} \in \mathrm{C}$ has, the left hand side of Eq. (4.4) has to be real because the right hand side of Eq. (4.4) is always real since $(\bar{q}, \bar{\nu}, \bar{B}) \in \mathbb{R}$. This condition together with the mixing of real and complex quantities in Eq. (4.4) is essential to understand the above described appearance of different sets of solutions.

As long as $B_{\mathrm{sub}} \geqslant B_{\mathrm{c}, 1}$ all solutions of the dispersion relation with $q=q_{\mathrm{m}}$ are real, i.e., $-i \omega=\omega_{2} \in \mathbb{R}$ (see Fig. 10). Using this result it follows from Eq. (4.4) that there is a value $\bar{\omega}_{2}=-\bar{\nu} \bar{q}_{\mathrm{m}}^{2}$ beyond which the radicand becomes negative. Since a complex value for the left hand side of Eq. (4.4) is not allowed, the solution does not exist beyond $\bar{\omega}_{2}=$ $-\bar{\nu} \bar{q}_{\mathrm{m}}^{2}$. This corresponds to the point in Fig. 10, where one of the solutions suddenly terminates at $B_{c, 2}$. Therefore the second critical induction yields

$$
\bar{B}_{c, 2}\left[\bar{\nu}, \bar{q}_{\mathrm{m}}(\bar{\nu}), \bar{\omega}_{2}=-\bar{\nu} \bar{q}_{\mathrm{m}}^{2}\right]=\sqrt{\frac{1}{2}\left(\frac{1}{\bar{q}_{\mathrm{m}}}+\bar{q}_{\mathrm{m}}+\bar{\nu}^{2} \bar{q}_{\mathrm{m}}^{2}\right)} .
$$

The first critical induction is the minimal induction for which real solutions exist, thus

$$
\begin{aligned}
\bar{B}_{c, 1}[ & \left.\bar{\nu}, \bar{q}_{\mathrm{m}}(\bar{\nu}), \bar{\omega}_{2, \mathrm{~min}}\right] \\
& =\left\{\frac{1}{2}\left(\frac{1}{\bar{q}_{\mathrm{m}}}+\bar{q}_{\mathrm{m}}\right)-2 \bar{\nu}^{2} \bar{q}_{\mathrm{m}}^{2}\left[\sqrt{1+D}-\left(1+\frac{D}{2}\right)^{2}\right]\right\}^{1 / 2},
\end{aligned}
$$

with $\quad \bar{\omega}_{2, \min }=\bar{\nu} \bar{q}_{\mathrm{m}}^{2} D=\left(\bar{\nu} \bar{q}_{\mathrm{m}}^{2} / 36\right)\left[-60+(108+12 \sqrt{93})^{2 / 3}\right.$ $\left.+144(108+12 \sqrt{93})^{-2 / 3}\right]$. Finally, the third critical induction is defined by $\bar{\omega}_{2}=0$ which leads to

$$
\bar{B}_{c, 0}\left[\bar{q}_{\mathrm{m}}(\bar{\nu}), \bar{\omega}_{2}=0\right]=\sqrt{\frac{1}{2}\left(\frac{1}{\bar{q}_{\mathrm{m}}}+\bar{q}_{\mathrm{m}}\right)} .
$$

The three thresholds (4.5)-(4.7) follow the relation $\bar{B}_{c, 1}$ $\leqslant \bar{B}_{c, 0} \leqslant \bar{B}_{c, 2}$, where the equal sign applies to $\bar{\nu}=0$. With increasing viscosity $\bar{\nu}$ the differences between the thresholds increase.

Eventually, we focus on the type of the prevailing bifurcation. In Fig. 11, the eigenvalue $-i \omega$ is plotted in the complex plane for both the viscous (a) and the inviscid (b) fluid. For the viscous fluid, the complex eigenvalues merge on the real axis at $B_{c, 1}$ and are real for all inductions larger than $B_{c, 1}$, as presented in Fig. 11(a). The crossing towards the positive real axis of one of the real eigenvalues occurs at $B_{c, 0}$. That defines the steady state character of the bifurcation.

With vanishing viscosity the character of the bifurcation approaches the splitting type $[9,10]$ where two pure imaginary eigenvalues split into two real ones, as shown in Fig. 11(b).

\section{DISCUSSION AND CONCLUSION}

Comparing the theoretical curves in Fig. 5 and the measured results, we find an excellent agreement for the frequency of the oscillating liquid ridges. Such an agreement is based on the fact that the viscosity and the finite depth of the fluid layer are taken into account. The only fit parameter is the permeability of the magnetic fluid. We obtained $\mu_{r}$ $=1.94$, which is slightly higher than the value 1.8 given by the producer. This is well justified by the increased mass density: It is nearly $8 \%$ higher than the one given in the data sheets of the producer, probably through evaporation of the kerosene. Therefore the contribution of the magnetite nanoparticles to the properties of the whole fluid becomes larger and the relative permeability increases.

The comparison of theoretical and experimental results for the case of the propagation velocity in a layer of $2 \mathrm{~mm}$ depth as presented in Fig. 7 is less successful. The measured values, which are denoted by open squares, are about $10 \%$ smaller than the theoretical prediction given by the dotted line. However, a convincing fit is presented by the dashed line, which gives the theoretical curve for a permeability of $\mu_{\mathrm{r}}=1.98$ and a layer thickness of $h=1.15 \mathrm{~mm}$. The permeability has slightly increased by $2 \%$ compared to the measurements in Fig. 5, probably due to evaporation during two months of measurements. 

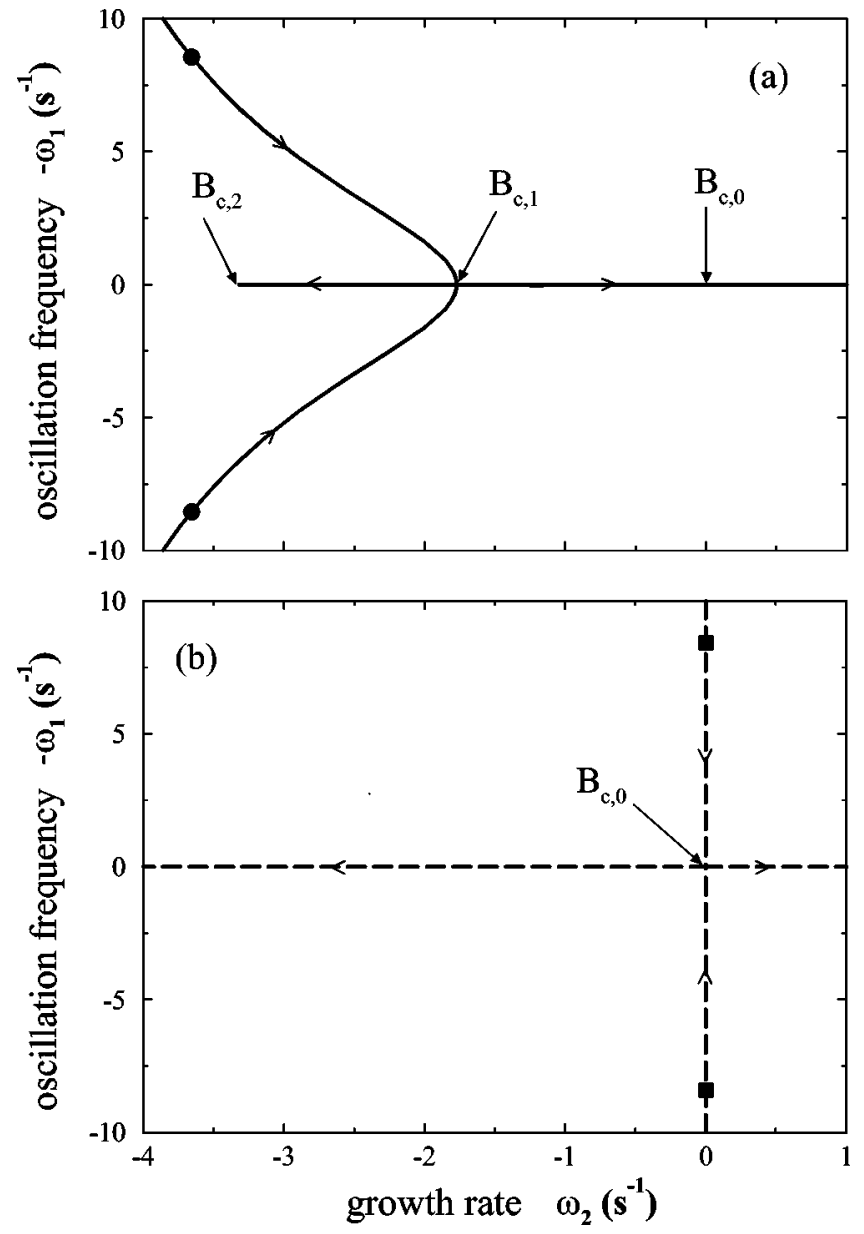

FIG. 11. Plot of the imaginary versus the real part of the complex eigenvalues $-i \omega=\omega_{2}-i \omega_{1}$ from Fig. 10 for the viscous (a) and inviscid (b) MF. (a) With increasing subcritical induction the complex eigenvalues pass through $B \simeq 16.960 \mathrm{mT}(\bullet)$ and merge at $B_{\mathrm{c}, 1} \simeq 17.006 \mathrm{mT}$. From there one negative real eigenvalue decreases until it terminates at $B_{\mathrm{c}, 2} \simeq 17.014 \mathrm{mT}$. The other real negative eigenvalue increases and changes its sign at $B_{\mathrm{c}, 0}$ $\simeq 17.009 \mathrm{mT}$. (b) The two imaginary eigenvalues pass through $B$ $\simeq 17.033 \mathrm{mT}(\boldsymbol{\square})$ and split at $B_{\mathrm{c}, 0} \simeq 17.059 \mathrm{mT}$ into one decreasing negative and one increasing positive real eigenvalue.

More puzzling is the drawdown of the fluid level to $h$ $=1.15 \mathrm{~mm}$, compared to the filling level of $2 \mathrm{~mm}$. Due to the inevitable field gradient at the edge of the vessel, some fluid is pulled from the center towards this edge. In this way the fluid level in the center is diminished, the area where the propagation velocity has been measured.

This behavior has been checked in independent measurements of the surface height of the static Rosensweig instability by means of a radioscopic method [15]. For the same type of MF, the same supercritical induction and a vessel depth of $4 \mathrm{~mm}$, a drawdown of the fluid level by a factor of 0.6 , was observed in the center of the dish. Thus, a reduction of the fluid depth by a factor of 0.58 , as suggested by the fit, is reasonable. Such a drawdown has drastic consequences for the already shallow depth of $2 \mathrm{~mm}$. The influence is less important for a reduction from $3 \mathrm{~mm}$ to $2 \mathrm{~mm}$, as demonstrated in Fig. 7(a) by the solid and dotted lines, respectively.

Figures 5(b) and 7(b) display the square of the measured quantities against the subcritical induction. It can be seen that the square-root-like dependence describes the frequency behavior with a precision of better than $10 \%$ within a range down to $80 \%$ of the critical induction.

The theoretical analysis of the bifurcation scenario makes it clear that oscillations almost up to the critical induction for the onset of the surface instability are to be expected. In fact, the interval from $B_{c, 1}$ to $B_{c, 0}$, where theory predicts a monotonous decay instead of an oscillatory decay, is estimated for our experimental parameters to be $0.003 \mathrm{mT}$ apart. This subtle difference between $B_{c, 0}$ and $B_{c, 1}$ cannot be resolved in our experiment. For the current experimental resolution, the viscosity of the magnetic fluid would have to be in the range of $1 \times 10^{-4} \mathrm{~m}^{2} \mathrm{~s}^{-1}$, but the viscosity of the used magnetic fluid EMG 909 is about two orders of magnitude smaller. The closeness to the inviscid case is further illustrated by the curves of oscillation frequency for the viscous and inviscid case, as displayed in Fig. 5, which can hardly be discriminated. Thus the experimental resolution in the studied system suggests a splitting type bifurcation, where the theoretical analysis unveils the true steady state character of the bifurcation.

According to the similar bifurcation scenario in the Faraday experiment $[16,17]$, a similar scaling behavior should also be observable for the natural frequency of periodic driven capillary-gravity waves. In close analogy, it can be initiated by a jump from supercritical to subcritical driving amplitude.

To conclude, we have experimentally investigated the decay of transient magnetic liquid ridges emerging after a jumplike increase of the magnetic induction with the help of a pulse sequence. It was possible to observe a dramatic decrease of the oscillation frequency and the propagation velocity in the vicinity of the bifurcation point. The scaling of both quantities agree in an excellent manner with the theoretical predictions, if the viscosity and the finite thickness of the magnetic liquid layer are taken into account. In quintessence, we have uncovered the subcritical part of the bifurcation diagram in the neighborhood of a splitting type bifurcation.

\section{ACKNOWLEDGMENTS}

The authors would like to thank Andreas Engel, René Friedrichs, Edgar Knobloch, Konstantin Morozov, Wim van Saarloos, and Mark Shliomis for interesting and clarifying discussions. The work was supported by the Deutsche Forschungsgemeinschaft under Grants Nos. Ri 1054/1-2 and La $1182 / 2-2$. 
[1] M.C. Cross and P.C. Hohenberg, Rev. Mod. Phys. 65, 851 (1993).

[2] G.I. Taylor, Z. Angew. Math. Mech. 13, 147 (1933).

[3] G.J. Stroebel and W.H. Warner, J. Elast. 3, 185 (1973).

[4] G.I. Taylor and A.D. McEwan, J. Fluid Mech. 22, 1 (1965).

[5] M.D. Cowley and R.E. Rosensweig, J. Fluid Mech. 30, 671 (1967).

[6] R.E. Rosensweig, Ferrohydrodynamics (Cambridge University Press, Cambridge, 1985).

[7] A. Lange, B. Reimann, and R. Richter, Phys. Rev. E 61, 5528 (2000).

[8] R. Friedrichs and A. Engel, Eur. Phys. J. B 18, 329 (2000).

[9] C. Martel and E. Knobloch, Phys. Rev. E 56, 5544 (1997).
[10] E. Knobloch, A. Mahalov, and J.E. Morsden, Physica D 73, 49 (1994).

[11] T. Mahr, Ph.D. thesis, Otto-von-Guericke Universität Magdeburg, 1998.

[12] D. Salin, Europhys. Lett. 21, 667 (1993).

[13] A. Lange, Magnetohydrodynamics 39, 65 (2003).

[14] Here we denote also an induction below $B_{\text {sup }}$ but larger than $B_{c}$ as a subcritical induction in order to avoid an inflation of indices.

[15] R. Richter and J. Bläsing, Rev. Sci. Instrum. 72, 1729 (2001).

[16] See Fig. 69 in Ref. [1].

[17] J. Miles and D. Henderson, Annu. Rev. Fluid Mech. 22, 143 (1990). 